\title{
Coherent X-Ray Radiation Generated Near the Axis of the Beam of Relativistic Electrons in an Artificial Periodic Structure
}

\author{
S. V. Blazhevich ${ }^{a, * *}$, Yu. A. Drygina ${ }^{a}$, O. Yu. Shevchuk ${ }^{a}$, and A. V. Noskov ${ }^{b}$ * \\ ${ }^{a}$ Belgorod State University, Belgorod, 308015 Russia \\ ${ }^{b}$ Shukhov Belgorod State Technological University, Belgorod, 308012 Russia \\ *e-mail: noskovbupk@mail.ru \\ **e-mail: blazh@bsu.edu.ru
}

Received October 7, 2019; revised December 10, 2019; accepted December 11, 2019

\begin{abstract}
A theory of the coherent X-ray radiation produced by a beam of relativistic electrons in an artificial periodic structure and propagating along the electron-beam axis is developed. Expressions describing the spectral-angular densities of parametric X-ray radiation in the direction close to the electron velocity vector (FPXR) and of transition radiation (TR) and their interference are obtained in the Bragg scattering geometry. An analysis based on the obtained expressions for the spectral-angular density shows possibilities of their use to determine the optimal parameters of the experiment recording the FPXR. The advantages of the experiment recording the FPXR at low electron energies $\left(E_{e} \leq 50 \mathrm{MeV}\right)$ are shown.
\end{abstract}

Keywords: artificial periodic structure, parametric X-ray radiation, dynamic diffraction, beam of relativistic electrons

DOI: $10.1134 / \mathrm{S} 1027451020030222$

\section{INTRODUCTION}

The authors of [1] were the first to consider the coherent $X$-ray radiation of relativistic electrons in an artificial periodic structure in the approximation of dynamic diffraction theory in the form of contributions of parametric X-ray radiation (PXR) and diffracted transition radiation (DTR). PXR in an artificial periodic structure is produced as a result of the diffraction of pseudophotons of the Coulomb field of relativistic electrons by target layers analogously to PXR generation in a single crystal as a result of diffraction at a system of parallel atomic planes $[2,3]$. DTR is a consequence of the diffraction of photons of transition radiation generated at the input target surface at target layers by analogy with DTR in a single crystal [4-6]. The dynamic theory of the radiation of relativistic electrons in periodic layered media [1] describes the experimental data given in [7] well. It is necessary to note that the radiation process in periodic layered media was traditionally considered in the Bragg scattering geometry and only for the particular case of symmetric reflection of the electron field with respect to the target surface where the angle between the target surface and reflecting planes was zero. Such consideration was also performed in [1]. In the general case of asymmetric reflection in the Bragg scattering geometry, the process of the coherent X-ray radiation of a relativistic electron in a periodic layered structure was first considered in [8], and then it was considered for beams of relativistic electrons in [9]. The manifestation of dynamic diffraction effects in the coherent $\mathrm{X}$-ray radiation of relativistic electrons propagating through an artificial periodic structure in the Laue scattering geometry was analyzed in detail in [10].

The theory of relativistic particle PXR in a single crystal predicts radiation not only near the Braggscattering direction, but also near the particle-velocity direction (forward PXR (FPXR)) [11, 12]. In the general case of the reflection of an electron Coulomb field that is asymmetric with respect to the target surface, in the Laue scattering geometry, the theoretical description of relativistic-electron FPXR in a single crystal was presented in [13]. For the case of symmetric reflection in the Bragg scattering geometry, a detailed theoretical description of relativistic-electron FPXR in a single crystal was given in [14]. FPXR has only been discovered experimentally in a single crystal and only in the Laue scattering geometry [15].

It is necessary to note that the FPXR of relativistic electrons in a periodic layered medium has not been observed experimentally. FPXR is the dynamic effect, and its experimental recording is very important for the dynamic theory of coherent $\mathrm{X}$-ray radiation.

In this paper, for the general case of electron-field reflection which is asymmetric with respect to the target surface, we consider the coherent $\mathrm{X}$-ray radiation 


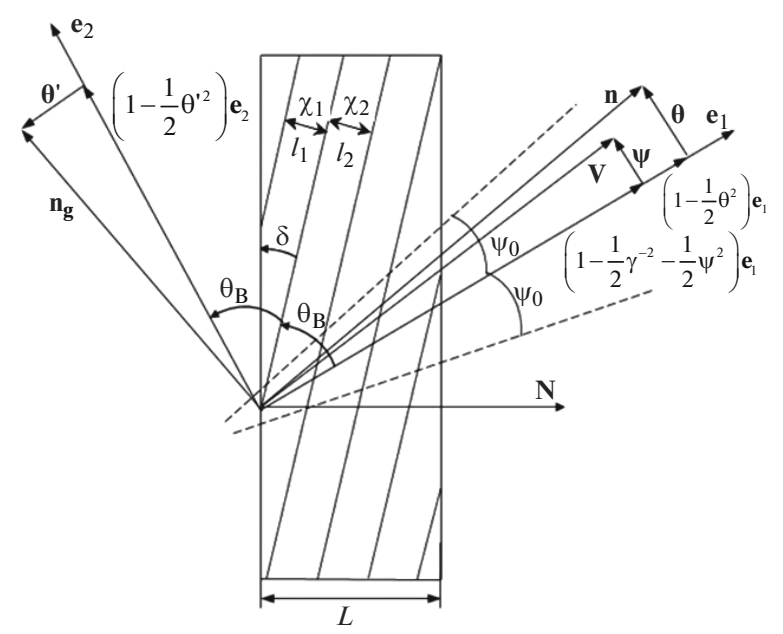

Fig. 1. Geometry of the radiation process.

of a beam of relativistic electrons in a periodic layered medium in the direction near that of the beam axis in the Bragg scattering geometry. We obtain and study expressions describing the spectral-angular radiation density.

\section{GEOMETRY OF THE RADIATION PROCESS}

We consider a beam of relativistic electrons propagating through a periodic structure in the Bragg scattering geometry (Fig. 1); it consists of alternating layers with the thicknesses $l_{1}$ and $l_{2}$, and permittivities $\chi_{1}$ and $\chi_{2}$, respectively ( $T=l_{1}+l_{2}$ is the period of the layered target). The reflecting layers were located at an angle $\delta$ to the target surface (Fig. 1), which corresponds to the case of asymmetric reflection of the radiation field ( $\delta=0$ is the particular case of symmetric reflection). We introduce the angular variables $\psi$, $\boldsymbol{\theta}^{\prime}$, and $\boldsymbol{\theta}$ in accordance with the definitions of the relativistic-electron velocity $\mathbf{V}$ and unit vector $\mathbf{n}$ (in the direction of momentum of the photon emitted near the direction of the electron velocity vector) and $\mathbf{n}_{\mathbf{g}}$ (in the Bragg scattering direction):

$$
\begin{gathered}
\mathbf{V}=\left(1-\frac{1}{2} \gamma^{-2}-\frac{1}{2} \psi^{2}\right) \mathbf{e}_{1}+\boldsymbol{\psi}, \quad \mathbf{e}_{1} \boldsymbol{\psi}=0, \\
\mathbf{n}=\left(1-\frac{1}{2} \theta^{2}\right) \mathbf{e}_{1}+\boldsymbol{\theta}, \quad \mathbf{e}_{1} \boldsymbol{\theta}=0, \quad \mathbf{e}_{1} \mathbf{e}_{2}=\cos 2 \theta_{\mathrm{B}}, \\
\mathbf{n}_{\mathbf{g}}=\left(1-\frac{1}{2} \theta^{\prime 2}\right) \mathbf{e}_{2}+\boldsymbol{\theta}^{\prime}, \quad \mathbf{e}_{2} \boldsymbol{\theta}=0,
\end{gathered}
$$

where $\boldsymbol{\theta}^{\prime}$ is the angle of coherent $\mathrm{X}$-ray radiation (PXR and DTR) in the Bragg scattering direction measured from the radiation-detector axis $\mathbf{e}_{2}, \boldsymbol{\psi}$ is the angle of deflection of the considered electron in the beam measured from an electron-beam axis $\mathbf{e}_{1}, \boldsymbol{\theta}$ is the angle of coherent X-ray radiation near the relativistic-electron velocity direction (FPXR and TR), and $\gamma=1 / \sqrt{1-V^{2}}$ is the electron Lorentz factor. The angular variables are considered in the form of the sum of components parallel and perpendicular to the figure plane: $\boldsymbol{\theta}=\boldsymbol{\theta}_{\|}+\boldsymbol{\theta}_{\perp}, \boldsymbol{\psi}=\boldsymbol{\psi}_{\|}+\boldsymbol{\psi}_{\perp}$. We consider FPXR and TR in the direction of the vector $\mathbf{n}$ (Fig. 1). We call the angle $\psi_{0}$ the initial divergence of the beam of radiating electrons (Fig. 1). The angle $\psi_{0}$ determines a cone limiting the portion of the electron beam beyond the limits of which the density of electrons decreases by a factor of $e$ or more as compared with that at the beam axis.

\section{SPECTRAL-ANGULAR RADIATION DENSITY}

When solving the problem, we consider the equation for the Fourier transform $\mathbf{E}(\mathbf{k}, \omega)=$ $\int d t d^{3} r \mathbf{E}(\mathbf{r}, t) \exp (i \omega t-i \mathbf{k r})$ of the electromagnetic field produced by an electron in a periodic layered medium; it follows from the Maxwell system of equations:

$$
\begin{aligned}
& \left(k^{2}-\omega^{2}\left(1+\chi_{0}\right)\right) \mathbf{E}(\mathbf{k}, \omega)-\mathbf{k}(\mathbf{k} \mathbf{E}(\mathbf{k}, \omega)) \\
& -\omega^{2} \sum_{\mathbf{g}} \chi_{-\mathbf{g}}^{\prime} \mathbf{E}(\mathbf{k}+\mathbf{g}, \omega)=4 \pi i \omega \mathbf{J}(\mathbf{k}, \omega),
\end{aligned}
$$

where $\mathbf{J}(\mathbf{k}, \omega)=2 \pi e \mathbf{V} \delta(\omega-\mathbf{k V})$ is the Fourier transform of the current density of a radiating electron, $\chi_{0}(\omega)$ is the average permittivity of the periodic layered medium, and $\chi_{\mathrm{g}}$ and $\chi_{-\mathrm{g}}$ are the Fourier coefficients of the expansion of the periodic-structure permittivity in terms of the vector $\mathbf{g}$ :

$$
\begin{aligned}
& \chi(\omega, \mathbf{r})=\sum_{\mathbf{g}} \chi_{\mathbf{g}}(\omega) \exp (i \mathbf{g r}) \\
= & \sum_{\mathbf{g}}\left(\chi_{\mathbf{g}}^{\prime}(\omega)+i \chi_{\mathbf{g}}^{\prime \prime}(\omega)\right) \exp (i \mathbf{g r})
\end{aligned}
$$

where $\chi_{0}=\chi_{0}^{\prime}+i \chi_{0}^{\prime \prime}, \chi_{\mathrm{g}}=\chi_{\mathrm{g}}^{\prime}+i \chi_{\mathrm{g}}^{\prime \prime}$.

The vector $\mathbf{g}$ is analogous to the reciprocal lattice vector in the crystal; it is perpendicular to the target layers, and its length is $g=\frac{2 \pi}{T} n, n=0, \pm 1, \pm 2, \ldots$. In the case of $\chi_{-\mathrm{g}}=0$, expression (2) describes the electric field in a homogeneous amorphous medium. 
In the periodic structure under consideration, the quantities $\chi_{0}$ and $\chi_{\mathrm{g}}$ have the following form

$$
\begin{gathered}
\chi_{0}(\omega)=\frac{l_{1}}{T} \chi_{1}+\frac{l_{2}}{T} \chi_{2}, \\
\chi_{\mathrm{g}}(\omega)=\frac{\exp \left(-i g l_{1}\right)-1}{i g T}\left(\chi_{2}-\chi_{1}\right) .
\end{gathered}
$$

Expression (4) implies the relations used below:

$$
\begin{gathered}
\chi_{0}^{\prime}=\frac{l_{1}}{T} \chi_{1}^{\prime}+\frac{l_{2}}{T} \chi_{2}^{\prime}, \quad \chi_{0}^{\prime \prime}=\frac{l_{1}}{T} \chi_{1}^{\prime \prime}+\frac{l_{2}}{T} \chi_{2}^{\prime \prime}, \\
\operatorname{Re} \sqrt{\chi_{\mathrm{g}} \chi_{-\mathrm{g}}}=\frac{2 \sin \left(\frac{g l_{1}}{2}\right)}{g T}\left(\chi_{2}^{\prime}-\chi_{1}^{\prime}\right), \\
\operatorname{Im} \sqrt{\chi_{\mathrm{g}} \chi_{-\mathrm{g}}}=\frac{2 \sin \left(\frac{g l_{1}}{2}\right)}{g T}\left(\chi_{2}^{\prime \prime}-\chi_{1}^{\prime \prime}\right) .
\end{gathered}
$$

The influence of the material on radiation formation and propagation is determined only by $\chi_{0}(\omega)$ and $\chi_{\mathrm{g}}(\omega)$. In this case, near the front and rear target boundaries (at a thickness on the order of that of the largest layer), these values change along the boundary, because they change along the boundaries of layers with thicknesses $l_{1}$ and $l_{2}$, as they are cut off. But since coherent-radiation formation and scattering occur in a large number of layers, the changes in $\chi_{0}(\omega)$ and $\chi_{g}(\omega)$ under consideration near the target boundaries do not affect the spectral-angular radiation density in any way.

Because the electromagnetic field produced by a relativistic electron in the $\mathrm{X}$-ray frequency range is transverse, the electromagnetic waves $\mathbf{E}(\mathbf{k}, \omega)$ (incident) and $\mathbf{E}(\mathbf{k}+\mathbf{g}, \omega)$ (diffracted in a periodic layered medium) are determined by two amplitudes with different values of the transverse polarization

$$
\begin{gathered}
\mathbf{E}(\mathbf{k}, \omega)=E_{0}^{(1)}(\mathbf{k}, \omega) \mathbf{e}_{0}^{(1)}+E_{0}^{(2)}(\mathbf{k}, \omega) \mathbf{e}_{0}^{(2)}, \\
\mathbf{E}(\mathbf{k}+\mathbf{g}, \omega)=E_{\mathbf{g}}^{(1)}(\mathbf{k}, \omega) \mathbf{e}_{1}^{(1)}+E_{\mathbf{g}}^{(2)}(\mathbf{k}, \omega) \mathbf{e}_{1}^{(2)},
\end{gathered}
$$

where the vectors $\mathbf{e}_{0}^{(1)}$ and $\mathbf{e}_{0}^{(2)}$ are perpendicular to the vector $\mathbf{k}$, and the vectors $\mathbf{e}_{1}^{(1)}$ and $\mathbf{e}_{1}^{(2)}$ are perpendicular to the vector $\mathbf{k}_{\mathbf{g}}=\mathbf{k}+\mathbf{g}$. The vectors $\mathbf{e}_{0}^{(2)}, \mathbf{e}_{1}^{(2)}$ lie in the plane of the vectors $\mathbf{k}$ and $\mathbf{k}_{\mathbf{g}}$ ( $\pi$ polarization), and the vectors $\mathbf{e}_{0}^{(1)}$ and $\mathbf{e}_{1}^{(1)}$ are perpendicular to it ( $\sigma$ polarization). Within the framework of the two-wave approximation of dynamic diffraction theory, if (6) is taken into account, Eq. (2) reduces to the well-known system of equations [16]:

$$
\left\{\begin{array}{l}
\left(\omega^{2}\left(1+\chi_{0}\right)-k^{2}\right) E_{0}^{(s)}+\omega^{2} \chi_{-\mathrm{g}} C^{(s, \tau)} E_{\mathbf{g}}^{(s)}=8 \pi^{2} i e \omega \mathbf{e}_{\mathbf{0}}^{(s)} \mathbf{V} \delta(\omega-\mathbf{k V}) \\
\omega^{2} \chi_{\mathrm{g}} C^{(s, \tau)} E_{0}^{(s)}+\left(\omega^{2}\left(1+\chi_{0}\right)-k_{\mathrm{g}}^{2}\right) E_{\mathrm{g}}^{(s)}=0 .
\end{array}\right.
$$

The quantities $C^{(s)}$ and $P^{(s)}$ in system of equations (7) are defined as follows:

$$
\begin{gathered}
C^{(s, \tau)}=\mathbf{e}_{0}^{(s)} \mathbf{e}_{1}^{(s)}=(-1)^{\tau} C^{(s)}, \quad C^{(1)}=1, \\
C^{(2)}=\left|\cos 2 \theta_{\mathrm{B}}\right|, \quad \mathbf{e}_{\mathbf{0}}^{(1)} \mathbf{V}=\theta_{\perp}-\psi_{\perp}, \\
\mathbf{e}_{\mathbf{0}}^{(2)} \mathbf{V}=\theta_{\|}+\psi_{\|},
\end{gathered}
$$

where $\theta_{\mathrm{B}}$ is the angle between the electron-beam axis and the reflecting layers (the Bragg angle). The length of the reciprocal lattice vector is defined by the expression $g=2 \omega_{\mathrm{B}} \sin \theta_{\mathrm{B}} / V$, where $\omega_{\mathrm{B}}$ is the Bragg frequency. The system of equations (7) at $s=1$ and $\tau=2$ describes $\sigma$ polarized fields. At $s=2$, system (6) describes $\pi$-polarized fields; in this case, if $2 \theta_{\mathrm{B}}<\pi / 2$, then $\tau=2$, and, in the opposite case, $\tau=1$.

We consider the most interesting case where the electron path length in the plate $L_{e}=L / \sin \left(\theta_{\mathrm{B}}+\delta\right)$ is larger than the $\mathrm{X}$-ray wave extinction length in a periodic layered medium, i.e., $b^{(s)}=L_{e} / 2 L_{\mathrm{ext}}^{(s)}$, which is the condition for the manifestation of dynamic effects in the radiation. At the same time, we consider a periodic layered medium as a thin nonabsorbing target, in which the photon path length in the target $\left(L_{f} \approx L_{e}\right)$ is significantly smaller than the length of $\mathrm{X}$-ray wave absorption in the periodic layered medium $L_{a b s}=$ $(1+r) / \omega\left(\chi_{1}^{\prime \prime}+r \chi_{2}^{\prime \prime}\right)\left(r=l_{2} / l_{1}\right)$, i.e., $L_{f} / L_{a b s} \ll 1$. For the process of the coherent $X$-ray radiation of a beam of relativistic electrons, by analogy with [8], we obtain an expression describing the spectral-angular FPXR density:

$$
\begin{aligned}
& \omega \frac{d^{2} N_{\mathrm{FPXR}}^{(s)}}{d \omega d \Omega} \\
& =\frac{e^{2}}{\pi^{2}} \frac{\Omega^{(s) 2}}{\left(\gamma^{-2}+\left(\theta_{\perp}-\psi_{\perp}\right)^{2}+\left(\theta_{\|}-\psi_{\|}\right)^{2}-\chi_{0}^{\prime}\right)^{2}} R_{\mathrm{FPXR}}^{(s)}, \\
& R_{\mathrm{FPXR}}^{(s)}=R_{1}^{(s)}+R_{2}^{(s)}+R_{\mathrm{INT}}^{(s)} \text {, } \\
& R_{1}^{(s)}=\frac{1}{\Delta^{(s)}} \frac{\sin ^{2}\left(b^{(s)} \Delta_{1}^{(s)} / 2\right)}{\Delta_{1}^{(s) 2}}, \\
& R_{2}^{(s)}=\frac{1}{\Delta^{(s)}} \frac{\sin ^{2}\left(b^{(s)} \Delta_{2}^{(s)} / 2\right)}{\Delta_{2}^{(s) 2}},
\end{aligned}
$$




$$
R_{\mathrm{INT}}^{(s)}=\frac{-1}{\Delta^{(s)}} \frac{\cos \left(b^{(s)} \sqrt{\xi^{(s) 2}-\varepsilon} / \varepsilon\right)\left[\cos \left(b^{(s)} \sqrt{\xi^{(s) 2}-\varepsilon} / \varepsilon\right)-\cos \left(b^{(s)}\left(\sigma^{(s)}-\xi^{(s)} / \varepsilon\right)\right)\right]}{\Delta_{1}^{(s)} \Delta_{2}^{(s)}},
$$

where the notation is introduced:

$$
\begin{aligned}
& \Omega^{(1)}=\theta_{\perp}-\psi_{\perp}, \quad \Omega^{(2)}=\theta_{\|}-\psi_{\|}, \\
& \Delta_{1,2}^{(s)}(\eta)=\sigma^{(s)}-\frac{\xi^{(s)} \mp \sqrt{\xi^{(s) 2}-\varepsilon}}{\varepsilon}, \\
& \Delta^{(s)}(\eta)=\xi^{(s) 2}-\varepsilon+\varepsilon \sin ^{2} \frac{b^{(s)} \sqrt{\xi^{(s) 2}-\varepsilon}}{\varepsilon}, \\
& \xi^{(s)}(\omega)=\eta^{(s)}(\omega)+\frac{1+\varepsilon}{2 v^{(s)}}, \\
& \eta^{(s)}(\omega)=\frac{2 \pi^{2} n^{2}}{T^{2} \omega_{\mathrm{B}}} L_{\mathrm{ext}}^{(s)}\left(1-\frac{\omega}{\omega_{\mathrm{B}}}\left(1+\theta_{\|} \sqrt{\frac{T^{2} \omega_{\mathrm{B}}^{2}}{\pi^{2} n^{2}}-1}\right)\right), \\
& b^{(s)}=\frac{L_{e}}{2 L_{\mathrm{ext}}^{(s)}}, \quad L_{e}=\frac{L}{\sin \left(\theta_{\mathrm{B}}+\delta\right)}, \\
& L_{\mathrm{ext}}^{(s)}=\frac{1}{\omega} \frac{\pi n}{\left|\sin \left(\frac{\pi n}{1+r}\right)\right|\left|\chi_{2}^{\prime}-\chi_{1}^{\prime}\right| C^{(s)}}, \quad \omega_{\mathrm{B}}=\frac{\pi n}{T \sin \theta_{\mathrm{B}}}, \\
& \varepsilon=\frac{\sin \left(\theta_{\mathrm{B}}-\delta\right)}{\sin \left(\theta_{\mathrm{B}}+\delta\right)}, \quad \sigma^{(s)}=\frac{\pi n}{C^{(s)}\left|\chi_{2}^{\prime}-\chi_{1}^{\prime}\right|\left|\sin \left(\frac{\pi n}{1+r}\right)\right|} \\
& \times\left(\gamma^{-2}+\left(\theta_{\perp}-\psi_{\perp}\right)^{2}+\left(\theta_{\|}+\psi_{\|}\right)^{2}-\chi_{0}^{\prime}\right) \text {, } \\
& v^{(s)}=\frac{C^{(s)} \mid \sin \left(\frac{\pi n}{1+r}\right)}{\frac{\pi n}{1+r}}\left|\frac{\chi_{2}^{\prime}-\chi_{1}^{\prime}}{\chi_{1}^{\prime}+r \chi_{2}^{\prime}}\right|, \\
& \chi_{0}^{\prime}=\frac{\chi_{1}^{\prime}+r \chi_{2}^{\prime}}{1+r}, \quad r=\frac{l_{2}}{l_{1}} \text {. }
\end{aligned}
$$

reflection where $\delta=0$, the asymmetry parameter $\varepsilon=1$. In Fig. 1, the positive direction of the angle $\delta$ is indicated.

The parameter $v^{(s)}$, taking values in the interval $0 \leq v^{(s)} \leq 1$, determines the degree of electron-field reflection from the target layers; this is due to the character of the interference of waves reflected from different layers, namely, either constructive $\left(v^{(s)} \approx 1\right)$ or destructive $\left(v^{(s)} \approx 0\right)$ one.

Spectral function $R_{\mathrm{FPXR}}^{(s)}(9 \mathrm{~b})$ is represented in the form of terms describing the contributions of two branches of X-ray waves $R^{(1)}$ and $R^{(2)}$, and also of their interference term $R_{\mathrm{INT}}^{(s)}$ to the FPXR spectrum. The wave vectors, whose lengths have the form

$$
\begin{aligned}
k^{(1,2)}= & \omega \sqrt{1+\chi_{0}^{\prime}}+\omega \frac{C^{(s)}\left|\chi_{2}^{\prime}-\chi_{1}^{\prime}\right|\left|\sin \left(\frac{\pi n}{1+r}\right)\right|}{2 \pi n \varepsilon} \\
& \times\left(\xi^{(s)}(\omega) \mp \sqrt{\xi^{(s)}(\omega)^{2}-\varepsilon}\right) .
\end{aligned}
$$

correspond to each X-ray wave. Expressions (11) follow from the solution of the dispersion equation $\left(\omega^{2}\left(1+\chi_{0}\right)-k^{2}\right)\left(\omega^{2}\left(1+\chi_{0}\right)-k_{\mathrm{g}}^{2}\right)-\omega^{4} \chi_{-\mathrm{g}} \chi_{\mathrm{g}} C^{(s) 2}=0$, following from (7).

The contributions of the first $R^{(1)}$ and second $R^{(2)}$ FPXR branches are significant in the case where the corresponding equations

$$
\begin{gathered}
\sigma^{(s)}-\frac{\xi^{(s)}-\sqrt{\xi^{(s) 2}-\varepsilon}}{\varepsilon}=0, \\
\sigma^{(s)}-\frac{\xi^{(s)}+\sqrt{\xi^{(s) 2}-\varepsilon}}{\varepsilon}=0,
\end{gathered}
$$

have solutions. Because the parameter $\sigma^{(s)}>1$, it is possible to show that Eq. (12b) has a solution always, and Eq. (12a) is solvable only under the condition $\varepsilon<1 / \sigma^{(s) 2}$. The solutions of Eqs. (12a) and (12b) determine the frequency in the vicinity of which the spectrum of FPXR photons emitted at a fixed observation angle is concentrated. It follows from Eq. (12) that the FPXR-spectrum maximum is always located beyond the total reflection (extinction) region: $\xi^{(s)}(\omega)=\sqrt{\varepsilon}+\left(\sigma^{(s)} \sqrt{\varepsilon}-1\right)^{2} / 2 \sigma^{(s)}>\sqrt{\varepsilon}$. In this frequency range, the length of the wave vector takes complex values even in the absence of absorption: the rad- 
icand in (11) is negative. The total reflection region is defined by the inequality:

$$
\begin{aligned}
-\sqrt{\varepsilon} & <\xi^{(s)}(\omega)<\sqrt{\varepsilon}, \\
-\sqrt{\varepsilon}-(1+\varepsilon) / 2 v^{(s)} & <\eta^{(s)}(\omega)<\sqrt{\varepsilon}-(1+\varepsilon) / 2 v^{(s)},
\end{aligned}
$$

from which it is seen that the width of this region is determined by $2 \sqrt{\varepsilon}$.

We obtained an expression describing the spectralangular density of the transition radiation:

$$
\begin{gathered}
\omega \frac{d^{2} N_{\mathrm{TR}}^{(s)}}{d \omega d \Omega}=\frac{e^{2}}{\pi^{2}} \Omega^{(s) 2}\left(\frac{1}{\gamma^{-2}+\left(\theta_{\perp}-\psi_{\perp}\right)^{2}+\left(\theta_{\|}-\psi_{\|}\right)^{2}}-\frac{1}{\gamma^{-2}+\left(\theta_{\perp}-\psi_{\perp}\right)^{2}+\left(\theta_{\|}-\psi_{\|}\right)^{2}-\chi_{0}^{\prime}}\right)^{2} R_{\mathrm{TR}}^{(s)} \\
R_{\mathrm{TR}}^{(s)}=1+\frac{\xi^{(s) 2}-\varepsilon}{\Delta^{(s)}(\eta)}\left[1-\frac{1}{\sqrt{\xi^{(s) 2}-\varepsilon}}\left(\left(\xi^{(s)}+\sqrt{\xi^{(s) 2}-\varepsilon}\right) \cos \left(b^{(s)} \Delta_{1}^{(s)}(\eta)\right)-\left(\xi^{(s)}-\sqrt{\xi^{(s) 2}-\varepsilon}\right) \cos \left(b^{(s)} \Delta_{2}^{(s)}(\eta)\right)\right)\right] .
\end{gathered}
$$

Expression (14) is valid for all possible values of $\xi^{(s)}(\omega)$ and differs significantly from the formula for TR from an amorphous plate with the same thickness $L$. This difference is caused by dynamic diffraction effects. It is significant only in the neighborhood of the Bragg frequency $\left|\xi^{(s)}(\omega)\right| \leq \varepsilon^{1 / 2}$. Beyond the region $\left|\xi^{(s)}(\omega)\right| \gg \varepsilon^{1 / 2}$, the spectral function takes the form of the well-known expression for the TR in an amorphous dielectric plate

$$
R_{\mathrm{TR}}^{(s)}=2\left(1-\cos \left(b^{(s)} \sigma^{(s)}\right)\right) .
$$

It follows from expression (15) that the destructive interference of TR waves emitted from the input and output target surfaces completely suppress frequencies that are far from the Bragg frequency under the resonance condition

$$
b^{(s)} \sigma^{(s)}=2 \pi m, m \text { is a natural number. }
$$

We obtain an expression describing the FPXR and TR interference:

$$
\begin{gathered}
=\frac{e^{2}}{\pi^{2}} \Omega^{(s) 2} \frac{\omega \frac{d^{2} N_{\mathrm{INT}}^{(s)}}{d \omega d \Omega}}{\gamma^{-2}+\left(\theta_{\perp}-\psi_{\perp}\right)^{2}+\left(\theta_{\|}-\psi_{\|}\right)^{2}-\chi_{0}^{\prime}} \\
\times\left(\frac{1}{\gamma^{-2}+\left(\theta_{\perp}-\psi_{\perp}\right)^{2}+\left(\theta_{\|}-\psi_{\|}\right)^{2}-\chi_{0}^{\prime}}\right. \\
\left.-\frac{1}{\gamma^{-2}+\left(\theta_{\perp}-\psi_{\perp}\right)^{2}+\left(\theta_{\|}-\psi_{\|}\right)^{2}}\right) R_{\mathrm{INT}}^{(s)}, \\
R_{\mathrm{INT}}^{(s)}=\frac{1}{2 \Delta^{(s)}(\eta)}\left[R_{\mathrm{FPXR} 1, \mathrm{TR}}^{(s)}-R_{\mathrm{FPXR} 2, \mathrm{TR}}^{(s)}\right],
\end{gathered}
$$

$$
\begin{gathered}
R_{\mathrm{FPXR} 1, \mathrm{TR}}^{(s)}=\frac{1}{\Delta_{1}^{(s)}(\eta)}\left[2\left(3 \sqrt{\xi^{(s) 2}-\varepsilon}+\xi^{(s)}\right)\right. \\
\times \sin ^{2}\left(\frac{b^{(s)} \Delta_{1}^{(s)}(\eta)}{2}\right)+\left(\xi^{(s)}-\sqrt{\xi^{(s) 2}-\varepsilon}\right) \\
\left.\times\left(\cos \left(2 b^{(s)} \sqrt{\xi^{(s) 2}-\varepsilon} / \varepsilon\right)+\cos \left(b^{(s)} \Delta_{2}^{(s)}(\eta)\right)\right)\right], \\
R_{\mathrm{FPXR} 2, \mathrm{TR}}^{(s)}=\frac{1}{\Delta_{2}^{(s)}(\eta)}\left[2\left(3 \sqrt{\xi^{(s) 2}-\varepsilon}-\xi^{(s)}\right)\right. \\
\times \sin ^{2}\left(\frac{b^{(s)} \Delta_{2}^{(s)}(\eta)}{2}\right)+\left(\xi^{(s)}+\sqrt{\xi^{(s) 2}-\varepsilon}\right) \\
\left.\times\left(\cos \left(2 b^{(s)} \sqrt{\xi^{(s) 2}-\varepsilon} / \varepsilon\right)-\cos \left(b^{(s)} \Delta_{1}^{(s)}(\eta)\right)\right)\right] .
\end{gathered}
$$

Expressions $R_{\mathrm{FPXR} 1, \mathrm{TR}}^{(s)}$ and $R_{\mathrm{FPXR} 2, \mathrm{TR}}^{(s)}$ describe the interference of the transition radiation with the first and second FPXR branches.

Expressions (9), (14), and (17) describing the contributions of FPXR and TR and their interference term to the spectral-angular radiation density of relativistic electrons with the inclusion of deviation of the direction of the electron velocity $\mathbf{V}$ from that of the electron-beam axis $\mathbf{e}_{1}$, given by the angle $\psi\left(\psi_{\perp}, \psi_{\|}\right)$, are the main result of this paper. These expressions are obtained for the general case of asymmetric electronfield reflection with respect to the target surface and contain a dependence on the asymmetry coefficient $\varepsilon$.

\section{NUMERICAL CALCULATIONS AND ANALYSIS OF THE SPECTRAL- ANGULAR RADIATION CHARACTERISTICS}

Using the obtained expressions (9), (14), and (17), we carry out numerical calculations for different parameters and analyze the properties of the coherent $\mathrm{X}$-ray radiation and the possibility of observing the FPXR of relativistic electrons generated in a periodic layered medium. For definiteness, we consider coherent radiation in a periodic layered medium consisting 


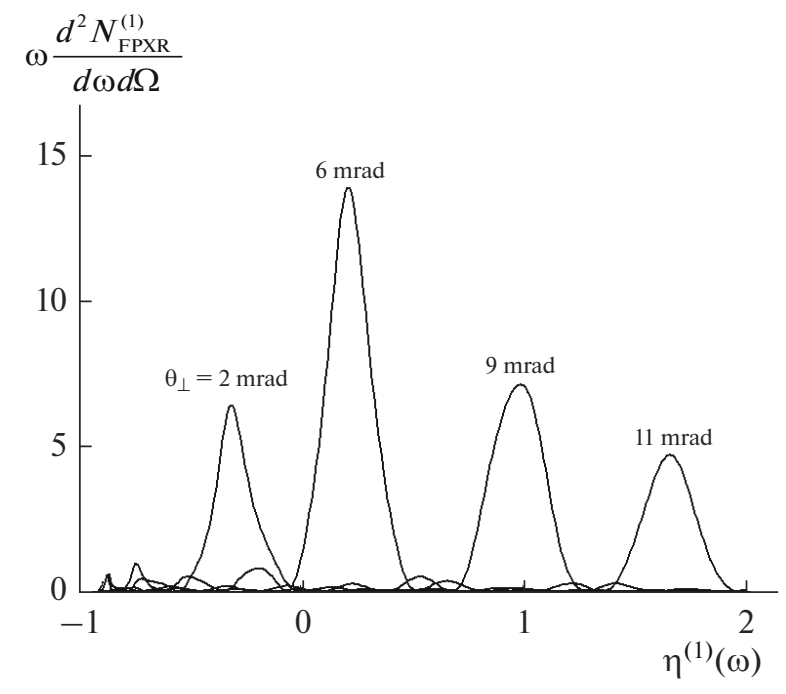

Fig. 2. Spectral-angular FPXR density. The parameters are $\theta_{\|}=0, \gamma=200, b^{(s)}=10$, and $\varepsilon=1$.

of layers with the permittivities $\chi_{1}^{\prime} \approx 10^{-4}$ and $\chi_{2}^{\prime} \approx 10^{-5}$, near the vicinity of the Bragg frequency $\omega_{B}$. We calculate the spectral-angular density of the coherent X-ray radiation for $\sigma$-polarized waves $(s=1)$ of the first harmonic $n=1$. We assume that the deviation of the electron velocity direction from the beam axis is zero $\psi=0 \quad\left(\psi_{\perp}=\psi_{\|}=0\right)$. The thicknesses of the reflecting layers are the same $r=l_{2} / l_{1}=1$; in this case, the average permittivity in the target is $\chi_{0}^{\prime}=-5.5 \times 10^{-5}$.

We consider the case where the inequality $\varepsilon>1 / \sigma^{(s) 2}$, is valid; i.e., only the second FPXR branch makes a contribution. Numerical calculations show that the inequality $\sigma^{(s)}>2$ holds for the parameters under consideration; in this case, the minimum asymmetry parameter for which there is no first FPXR branch is $\varepsilon=0.25$. It is necessary to note that, in the case of $\varepsilon<0.25$ and the parameters under consideration, the spectral-angular density of the second FPXR branch significantly exceeds that of the first branch. We write the expression describing the spectral-angular density of the second FPXR branch (9a) and (9d) for $\sigma$-polarized waves at $\psi_{\perp}=\psi_{\|}=0$

$$
\omega \frac{d^{2} N_{\mathrm{FPXR}}^{(1)}}{d \omega d \Omega}=\frac{e^{2}}{\pi^{2}} \frac{\theta_{\perp}^{2}}{\left(\gamma^{-2}+\theta_{\perp}^{2}+\theta_{\|}^{2}-\chi_{0}^{\prime}\right)^{2}} R_{2}^{(1)},
$$

$$
\begin{gathered}
R_{2}^{(1)}=\frac{1}{\xi^{(1) 2}-\varepsilon+\varepsilon \sin ^{2} \frac{b^{(1)} \sqrt{\xi^{(1) 2}-\varepsilon}}{\varepsilon}} \\
\times \frac{\sin ^{2}\left(\frac{b^{(1)}}{2}\left(\sigma^{(1)}-\frac{\xi^{(1)}+\sqrt{\xi^{(1) 2}-\varepsilon}}{\varepsilon}\right)\right)}{\left(\sigma^{(1)}-\frac{\xi^{(1)}+\sqrt{\xi^{(1) 2}-\varepsilon}}{\varepsilon}\right)^{2}} . \\
\xi^{(1)}(\omega)=\eta^{(1)}(\omega)+\frac{1+\varepsilon}{2 v^{(s)}},
\end{gathered}
$$

where

$$
\sigma^{(1)}=\frac{\pi}{\left|\chi_{2}^{\prime}-\chi_{1}^{\prime}\right|}\left(\gamma^{-2}+\theta_{\perp}^{2}+\theta_{\|}^{2}-\chi_{0}^{\prime}\right) .
$$

We consider the dependence of the spectral-angular FPXR density of relativistic electrons on the observation angle $\theta_{\perp}$; in this case, we assume that $\theta_{\|}=0$. Figure 2 shows curves constructed using formula (18a) and which describe the spectral-angular FPXR densities for different observation angles at $\gamma=200$. The function describing the angular FPXR density $F^{(1)}=\frac{e^{2}}{\pi^{2}} \frac{\theta_{\perp}^{2}}{\left(\gamma^{-2}+\theta_{\perp}^{2}+\theta_{\|}^{2}-\chi_{0}^{\prime}\right)^{2}}$ has a maximum at the point $\theta_{\perp}=\sqrt{\gamma^{-2}-\chi_{0}^{\prime}}$. For the parameters $\chi_{0}^{\prime}=-5.5 \times 10^{-5}$ and $\gamma=200$, under consideration, this angle is $\theta_{\perp} \approx 9 \mathrm{mrad}$, which is demonstrated by the curve given in Fig. 3. However, in the case under consideration, the angle of the maximum of the spectral-angular FPXR density is $\theta_{\perp}^{\max } \approx 6 \mathrm{mrad}$ (Fig. 2). This is related to the fact that the maximum of the dynamic spectral function $R_{2}^{(1)}$ increases with decreasing observation angle $\theta_{\perp}$, the curves given in Fig. 4 demonstrate this effect. It is important to note that the kinematic PXR theory does not predict the FPXR peak.

Figure 5 shows curves demonstrating an increase in the spectral-angular FPXR density with increasing electron path in the target $b^{(1)}=L_{e} / 2 L_{\mathrm{ext}}^{(1)}$, it is expressed in a double extinction length.

The obtained expression (18a) predicts the influence of the asymmetry of the electron-field reflection with respect to the target surface on the spectral-angular radiation density. We consider the dependence of the spectral-angular FPXR density on the reflection asymmetry at a fixed observation angle. The curves constructed using formulas (18a) and given in Fig. 6 demonstrate an increase in the amplitude of the spectral-angular FPXR density with decreasing asymmetry 


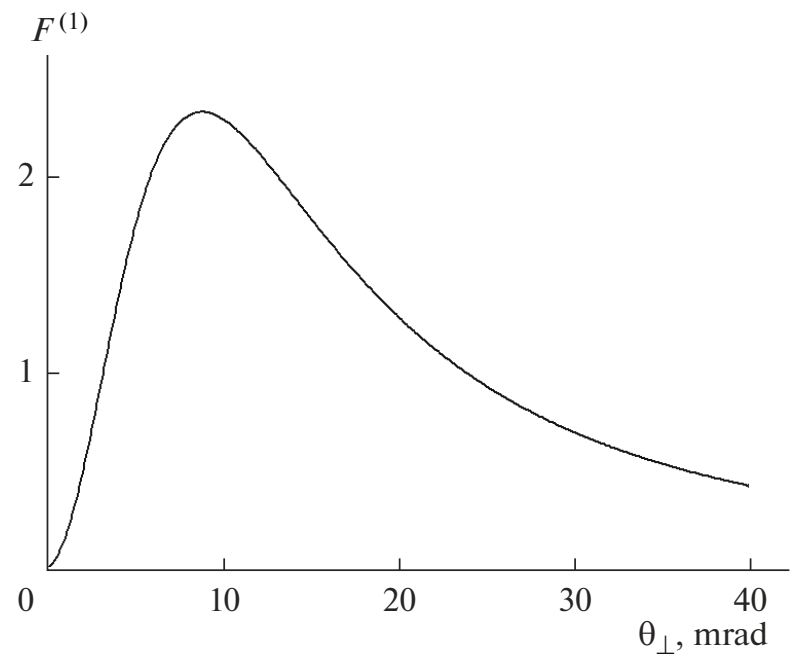

Fig. 3. Angular part of the spectral-angular FPXR density. The parameter are $\theta_{\|}=0, \gamma=200, b^{(s)}=10$, and $\varepsilon=1$.

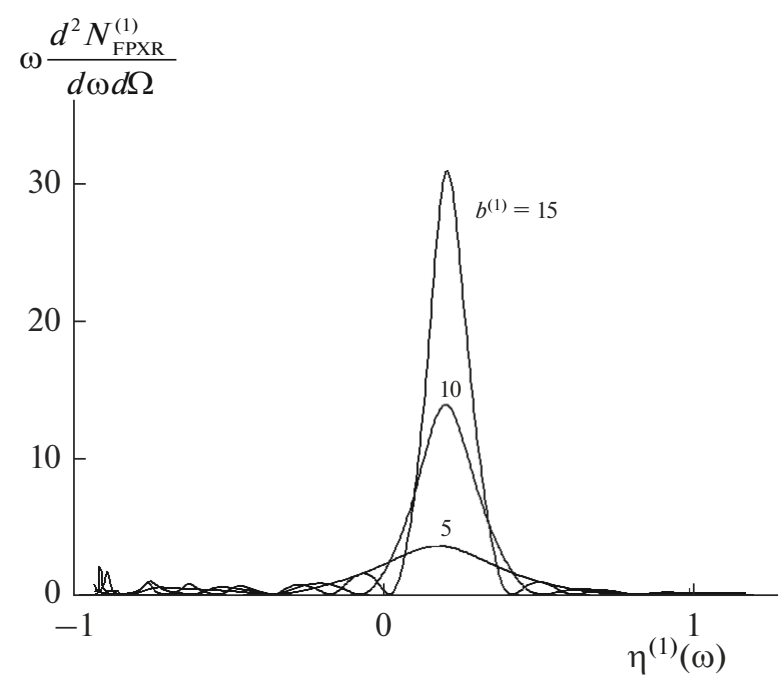

Fig. 5. Spectral-angular FPXR density for different electron paths in the target. The parameters are $\theta_{\perp}=6 \mathrm{mrad}$, $\theta_{\|}=0, \gamma=200$, and $\varepsilon=1$.

parameter $\varepsilon$, the spectral width decreases in this case. For example, $\varepsilon=0.5\left(\right.$ at $\theta_{\mathrm{B}}=10^{\circ}$ and $\delta=3.4^{\circ}$ ) and $\varepsilon=1.5\left(\right.$ at $\theta_{\mathrm{B}}=10^{\circ}$ and $\left.\delta=-2^{\circ}\right)$. The case of symmetric reflection corresponds to $\varepsilon=1(\delta=0)$.

In view of the fact that the transition radiation is the background when experimentally recording FPXR and studying its properties, it is necessary to analyze the spectral-angular TR density and the influence of the contribution of the FPXR and TR interference to

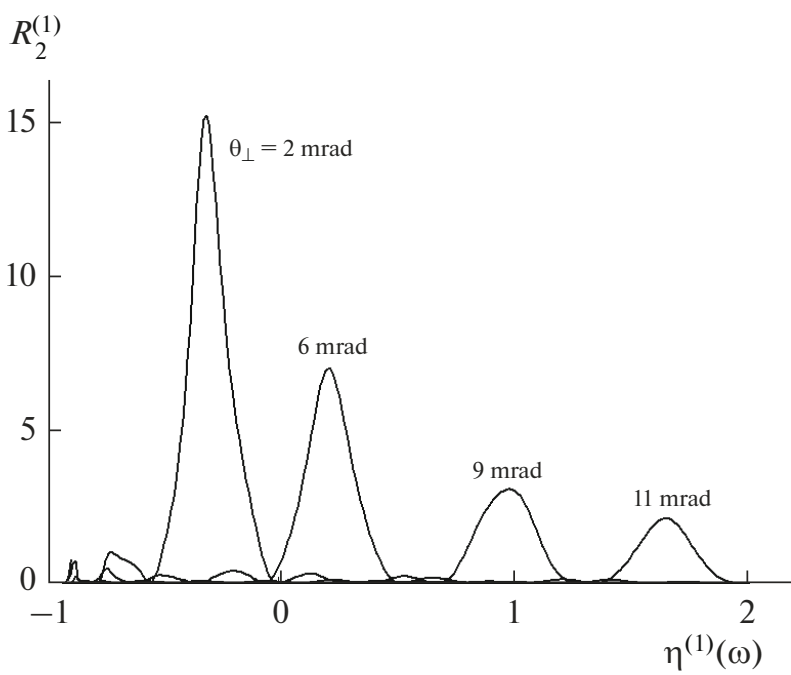

Fig. 4. FPXR spectrum. The parameters are $\theta_{\|}=0$, $\gamma=200, b^{(s)}=10$, and $\varepsilon=1$.

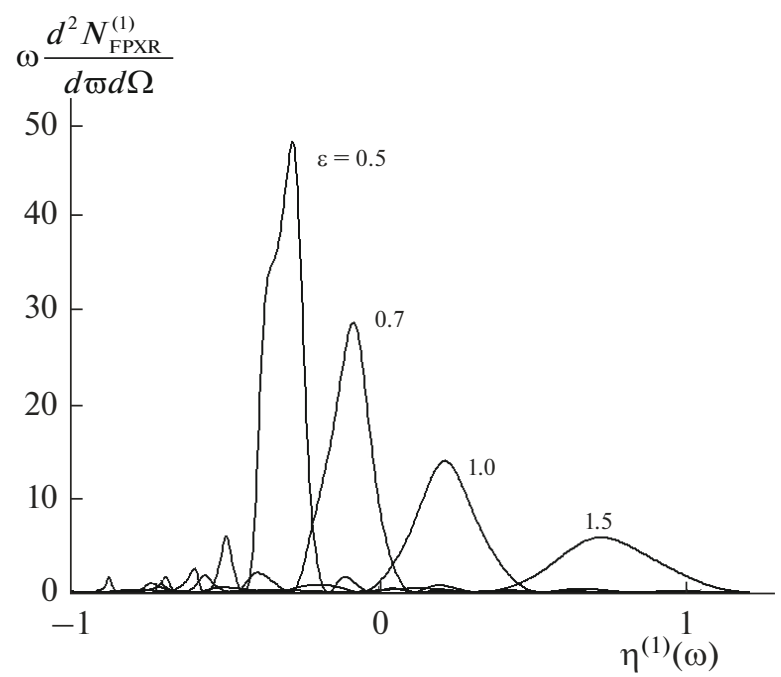

Fig. 6. Influence of the reflection asymmetry on the spectral-angular FPXR density for a fixed observation angle. The parameters are $\theta_{\perp}=6 \mathrm{mrad}, \theta_{\|}=0, \gamma=200$, and $b^{(s)}=10$.

the coherent X-ray radiation. Figure 7 shows the curves constructed using formula (14a) and describing the spectral-angular TR density for a fixed observation angle. The curves were constructed for different values of the parameter $b^{(1)}=L_{e} / 2 L_{\mathrm{ext}}^{(1)}$. The solid curve in Fig. 7 constructed at $b^{(1)}=10.86$, corresponds to resonance condition (16) at $m=7$. It is seen from the figure that a small increase in the parameter $b^{(1)}$ (in the 


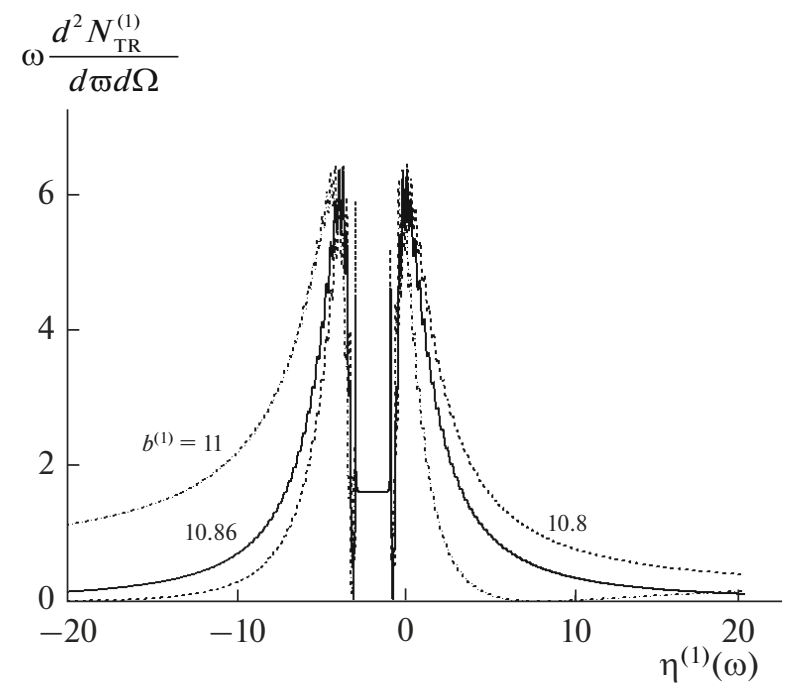

Fig. 7. Spectral-angular TR density for different $b^{(1)}$. The parameters are $\theta_{\perp}=6 \mathrm{mrad}, \theta_{\|}=0, \gamma=200$, and $\varepsilon=1$.

electron path in the target) leads to a decrease in the spectral-angular TR density on the right and to an increase in it on the left, which can be useful for experimental identification of the FPXR peak.

In some cases, the destructive interference of FPXR and TR waves can be significant. Figure 8 shows the curves constructed using formulas (18a), (14a), and (17a) and describing the spectral-angular densities of the FPXR and TR and their interference. It follows from the figure that, although the condition for suppression of the TR background is satisfied far from the Bragg frequency $\omega_{B}\left(\eta^{(1)}=0\right)$, the destructive interference of the FPXR and TR waves is, however, significant, which can make experimental FPXR recording difficult under these conditions.

Figure 9 shows curves, which are analogous to those in Fig. 8, but at $b^{(1)}=10$. The constructive interference of the FPXR and TR waves follows from Fig. 9, although TR waves with frequencies that are far from the Bragg frequency are not suppressed.

Figure 10 shows curves, which are analogous to those in Fig. 8, but in the asymmetric case at $\varepsilon=0.5$. A multiple increase in the spectral-angular FPXR density follows from the figure (compared with Fig. 8).

We consider the spectral-angular radiation density in the case of smaller energies of relativistic electrons (at $\gamma=100$ ). In the case under consideration, the angle of the maximum in the spectral-angular FPXR density is $\theta_{\perp}^{\max } \approx 9 \mathrm{mrad}$. Curves describing the spectralangular densities of the FPXR and TR and their interference are constructed in Figs. 11 and 12. In Fig. 11, the curves are constructed in the case of dominant constructive FPXR and TR interference. The FPXR

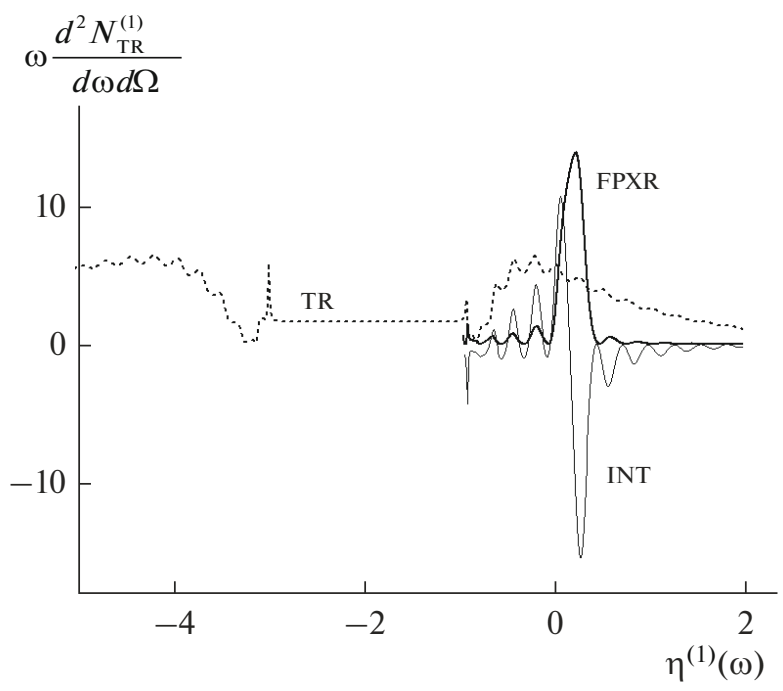

Fig. 8. Spectral-angular densities of the FPXR and TR and their interference. The parameters are $\theta_{\perp}=6 \mathrm{mrad}$, $\theta_{\|}=0, \gamma=200$, and $b^{(1)}=11$.

and TR interference is destructive in Fig. 12; however, the TR background is very small in the region of the FPXR peak. This background is suppressed because of destructive interference of the TR waves from the front and rear boundaries; i.e., condition (16) is satisfied. It is necessary to note that there is a significant decrease in the FPXR peak amplitude at $\gamma=100$, as compared with $\gamma=200$; however, the TR background decreases to a larger extent.

We consider the spectral-angular radiation densities in the case of smaller energies of relativistic electrons at $\gamma=50$. In this case, the angle of the maximum in the spectral-angular FPXR density is $\theta_{\perp}^{\max } \approx 12 \mathrm{mrad}$.

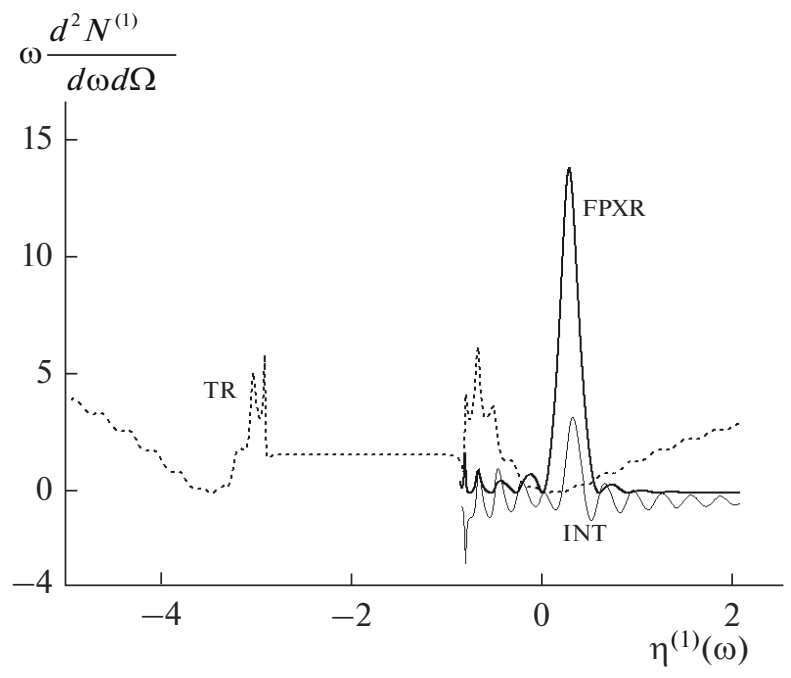

Fig. 9. The same as in Fig. 8, but at $b^{(1)}=10$. 


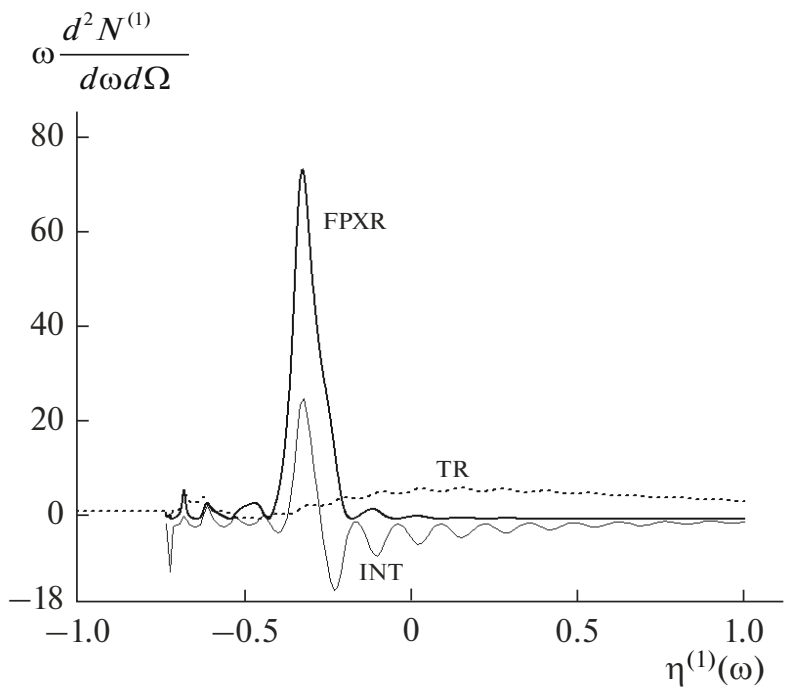

Fig. 10. Spectral-angular densities of the FPXR and TR and their interference. The parameters are $\theta_{\perp}=6 \mathrm{mrad}$, $\theta_{\|}=0, \gamma=200, b^{(1)}=11$, and $\varepsilon=0.5$.

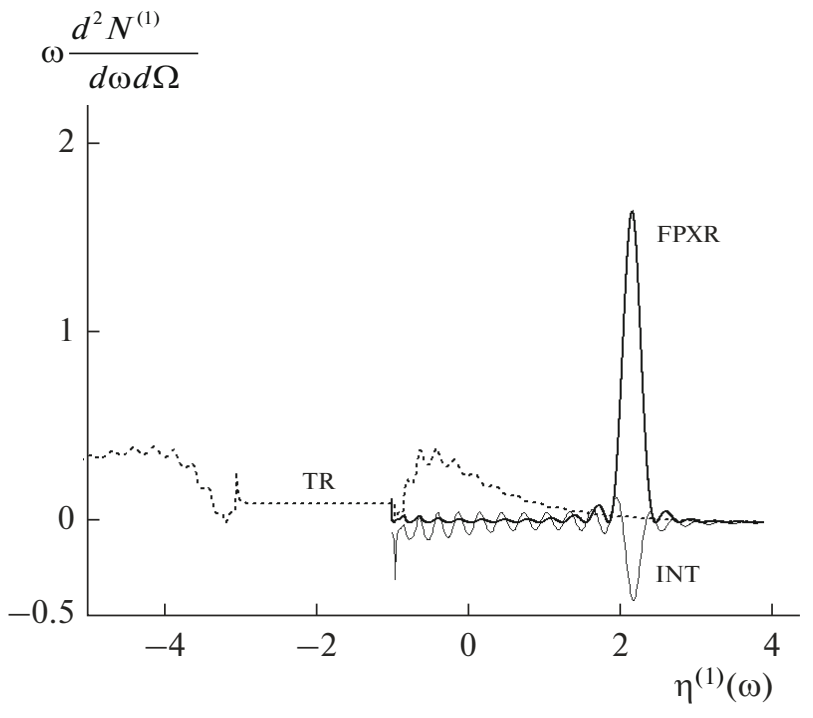

Fig. 12. The same as in Fig. 11 , but at $b^{(1)}=10$.

As was expected, an even larger decrease in the TR background with respect to the FPXR peak amplitude follows from Figs. 13 and 14.

If it is necessary, then formulas (9), (14), and (17) make it possible to average them over all possible rectilinear trajectories of electrons in the beam. For example, in the case of a Gaussian distribution of electrons in the beam $f(\psi)=\frac{1}{\pi \psi_{0}^{2}} \exp \left(-\frac{\psi_{\perp}^{2}+\psi_{\|}^{2}}{\psi_{0}^{2}}\right)$, the averaging of the spectral-angular densities has the form

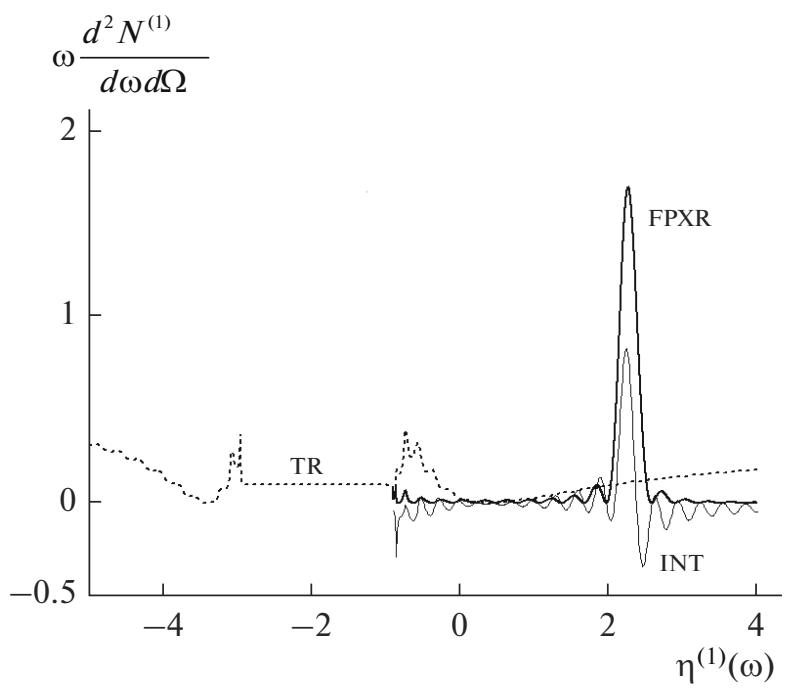

Fig. 11. Spectral-angular densities of the FPXR and TR and their interference. The parameters are $\theta_{\perp}=9 \mathrm{mrad}$, $\theta_{\|}=0, \gamma=100, b^{(1)}=10.2$, and $\varepsilon=1$.

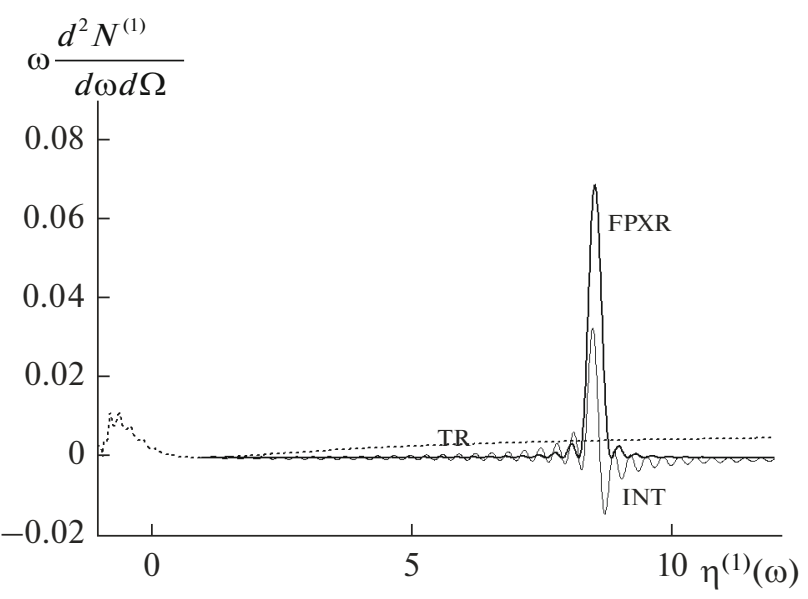

Fig. 13. Spectral-angular densities of the FPXR and TR and their interference. The parameters are $\theta_{\perp}=12 \mathrm{mrad}$, $\theta_{\|}=0, \gamma=50, b^{(1)}=10$, and $\varepsilon=1$.

$$
\begin{gathered}
\left\langle\omega \frac{d^{2} N_{\mathrm{FPXR}, \mathrm{TR}, \mathrm{INT}}^{(1)}}{d \omega d \Omega}\right\rangle=\frac{1}{\pi \psi_{0}^{2}} \\
\times \int_{-\infty}^{\infty} \int_{-\infty}^{\infty} \omega \frac{d^{2} N_{\mathrm{FPXR}, \mathrm{TR}, \mathrm{INT}}^{(1)}}{d \omega d \Omega} \exp \left(-\frac{\psi_{\perp}^{2}+\psi_{\|}^{2}}{\psi_{0}^{2}}\right) d \psi_{\perp} d \psi_{\|} .
\end{gathered}
$$

To take into account the multiple scattering of radiating electrons in the medium, it is possible to average the spectral-angular radiation densities over the angular distribution of electrons in the beam in the form of 


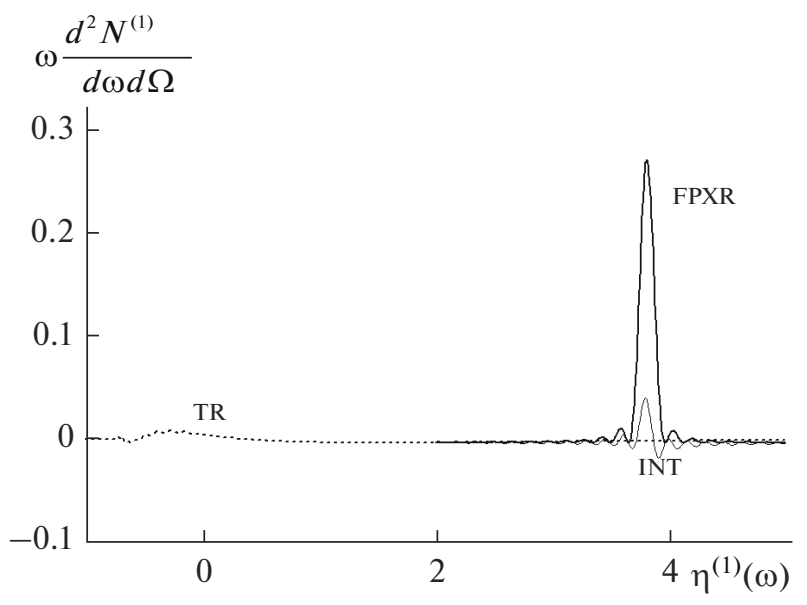

Fig. 14. The same as in Fig. 13, but in the asymmetric case, $\varepsilon=0.5$.

a Gaussian function varying with the path length in the target $t$ because of multiple electron scattering:

$$
f(\psi, t)=\frac{1}{\pi\left(\psi_{0}^{2}+\psi_{s}^{2} t\right)} \exp \left(-\frac{\psi_{\perp}^{2}+\psi_{\|}^{2}}{\psi_{0}^{2}+\psi_{s}^{2} t}\right),
$$

i.e., we average over a spreading beam of rectangular trajectories of radiating electrons at the electron path length in the target $L_{e}$. Here, $\psi_{s}^{2}=\frac{E_{s}^{2}}{m^{2} \gamma^{2}} \frac{1}{L_{R}}\left(1+0.038 \ln \left(\frac{t}{L_{R}}\right)\right)^{2}$ is the average square of the angle of multiple electron scattering per length unit [17], $E_{s} \approx \frac{4 \pi m^{2}}{e^{2}} \approx 21 \mathrm{MeV}$, and $L_{R}$ is the radiation length. In this case, the averaging of the spectralangular densities has the form

$$
\begin{gathered}
\left\langle\omega \frac{d^{2} N_{\mathrm{FPXR}, \mathrm{TR}, \mathrm{INT}}^{(1)}}{d \omega d \Omega}\right\rangle \\
=\frac{1}{\pi L_{e}} \int_{0}^{L_{e}} d t \int_{-\infty}^{\infty} \int_{-\infty}^{\infty} d \psi_{\perp} d \psi_{\|} \frac{\exp \left(-\frac{\psi_{\perp}^{2}+\psi_{\|}^{2}}{\psi_{0}^{2}+\psi_{s}^{2} t}\right)}{\psi_{0}^{2}+\psi_{s}^{2} t} \\
\times \omega \frac{d^{2} N_{\mathrm{FPXR}, \mathrm{TR}, \mathrm{INT}}^{(1)}}{d \omega d \Omega} .
\end{gathered}
$$

\section{CONCLUSIONS}

We have developed a dynamic theory of coherent $\mathrm{X}$-ray radiation generated by a beam of relativistic electrons in the direction near to the beam axis in a periodic layered medium. The radiation process was considered in the Bragg scattering geometry for the general case of asymmetric electron-field reflection with respect to the target surface. Based on the twowave approximation of dynamic diffraction theory, we obtained expressions describing the spectral-angular densities of the FPXR and TR and their interference term. We showed the possibility of observing FPXR. It was shown that the FPXR and TR interference can be constructive and destructive. A decrease in the asymmetry parameter $\varepsilon$ can lead to a significant increase in the amplitude of the spectral-angular FPXR density. A decrease in the energy of radiating electrons leads to that in the amplitude of the spectral-angular FPXR density; however, a significant decrease in the TR background is a positive moment in this case.

The performed analysis and the obtained expressions for the spectral-angular densities of the FPXR and TR (and for their interference) can be used to determine the optimal parameters of an experiment recording a FPXR beam of relativistic electrons propagating through a target made of a periodic layered material.

\section{REFERENCES}

1. N. N. Nasonov, V. V. Kaplin, S. R. Uglov, M. A. Piestrup, and C. K. Gary, Phys. Rev. E 68, 036504 (2003).

2. G. M. Garibyan and S. Yang, Sov Phys. JETP 34, 495 (1971).

3. V. G. Baryshevskii and I. D. Feranchuk, Sov Phys. JETP 34, 502 (1971).

4. V. G. Baryshevsky, Nucl. Instrum. Methods Phys. Res., Sect. A 122, 13 (1997).

5. X. Artru and P. Rullhusen, Nucl. Instrum. Methods Phys. Res., Sect. B 145, 1 (1998).

6. N. N. Nasonov, Phys Lett. A 246, 148 (1998).

7. V. V. Kaplin, S. R. Uglov, V. N. Zabaev, M. A. Piestrup, C. K. Gary, N. N. Nasonov, and M. K. Fuller, Appl. Phys. Lett. 76, 3647 (2000).

8. S. V. Blazhevich, Yu. P. Gladkikh, and A. V. Noskov, J. Surf. Invest.: X-Ray, Synchrotron Neutron Tech. 7 (2), 388 (2013).

9. S. V. Blazhevich and A. V. Noskov, J. Exp. Theor. Phys. 152 (2), 223 (2017).

10. S. V. Blazhevich, Yu. P. Gladkikh, and A. V. Noskov, J. Surf. Invest.: X-Ray, Synchrotron Neutron Tech. 8 (6), 1351 (2014).

11. G. M. Garibyan and S. Yang, Sov Phys. JETP 36, 631 (1973).

12. A. Caticha, Phys. Rev. B 45, 9541 (1992).

13. S. V. Blazhevich and A. V. Noskov, J. Exp. Theor. Phys. 109 (6), 901 (2009).

14. N. Nasonov and A. Noskov, Nucl. Instrum. Methods Phys. Res., Sect. B 201, 67 (2003).

15. A. N. Aleinik, A. N. Baldin, E. A. Bogomazova, I. E. Vnukov, et al., JETP Lett. 80, 393 (2004).

16. V. A. Bazylev and N. K. Zhevago, Radiation of Fast Particles in Matter and External Fields (Nauka, Moscow, 1987) [in Russian].

17. Particle Data Group, R. M. Barnett, et al., Phys. Rev. D: Part. Fields 54, 1 (1996).

Translated by L. Kulman 